inoculation of any bacterial vaccine. At $a$ we have represented in the form of a straight line the resisting power of the patient anterior to inoculation. Such a base line would represent in the case of an anti-typhoid inoculation the bactericidal power of the patient's blood anterior to inoculation and in the case of an anti-staphylococcus inoculation the phagocytic power of the blood as measured by Leishman's method; in the case of an anti-tuberculous inoculation, such as is here in question, the agglutinating power exerted by the blood on the test fluid which is here in question. At $b$ we have represented in the form of the descending limb
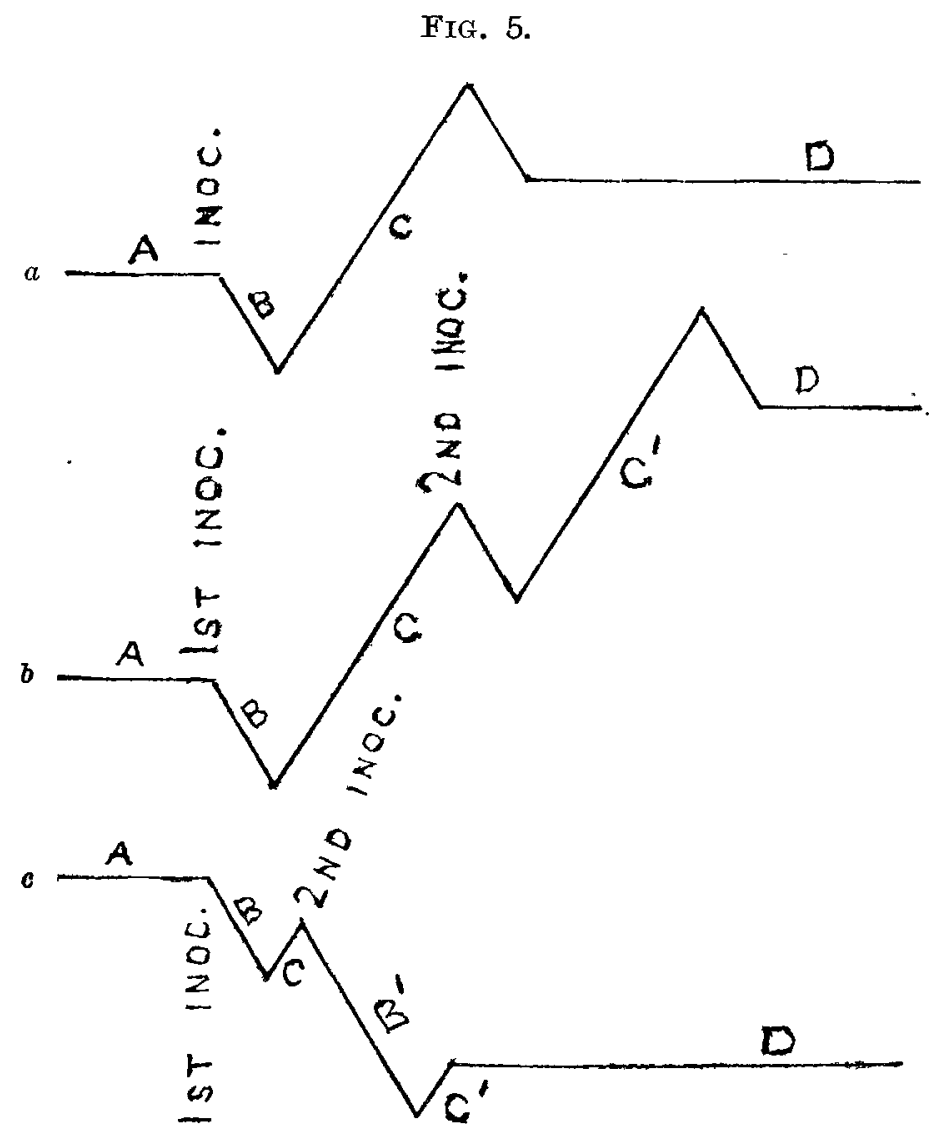

of the curve a decline in the resisting power of the organism such as follows, as I have endeavoured to show, upon every inoculation process. I have spoken of this as the "negative phase." At $c$ we have the ascending limb in the curve, representing an increased resistance to infection. I have spoken of this as the "positive phase." At $d$ we have the final result of a successful inoculation -a higher base line of resistance. In Fig. $5 b$ we have represented the event of two successive inoculations undertaken with appropriate doses of vaccine and appropriately interspaced. It will be seen that here the negative phase of the second inoculation in the figure comes on the top of the positive phase of the first inoculation, with the result that there is no such sinking away of the resistance below the original base line as occurred after the first inoculation. Let it be noted further that the effect of the two successive inoculations here undertaken is cumulative, and it is cumulative, as we desire it to be, in the direction of the positive phase and of increased resistance to bacterial invasion. In Fig. $5 c$, on the other hand, we have represented the event of two successive inoculations inappropriately interspaced. It will be seen that the second inoculation here falls upon the negative phase of the first inoculation, with the result that the resistance of the organism, already reduced by the first inoculation, is still further reduced. have shown elsewhere that when an undue tax is placedeither in this way or by a single inoculation of an excessive dose-on the responding powers of the organism that response may fail and the patient may after treatment continue, at any rate for a prolonged period, on a lower base line of resisting power.

The application of these general principles in connexion with the therapentic inoculation of Koch's new tuberculin appears to me to be important. It would seem to me that-as is now, I understand, done in Professor Koch's clinic
- every patient who is submitted to anti-tuberculous inoculation with the new tuberculin ought to be regularly tested by the serum test to ascertain that he responds to each successive inoculation. When such a system of tests is instituted we may hope to be successful in dealing with localised tuberculous infection by achieving a cumulative positive phase and in maintaining a higher base line of resistance. When the tests are omitted we may chance to achieve by our inoculations only a cumulative negative phase.

Lower Seymour-street, $W$.

\section{A NOTE ON THE POSITION OF THE HEART IN SOME CASES OF} AORTIC ANEURYSM.

By HUGH WALSHAM, M.D. CANTAB, F.R.C.P. LoND., ASSISTANT PHYSICIAN (FORMERIY PATHOLOGIST) TO THE CITY OF LONDON HOSPITAL FOR DISFASES OF THE CHEST, LONDON HOSPITAL FOR DISEASES
VICTORLA PARK.

IT would have been expected a priori that aneurysms springing from the arch of the aorta, especially when of large size, would cause considerable displacement of the heart downwards. This is found to be the case and has been insisted upon by most writers on the subject. This low position of the heart gives rise to well-marked epigastric pulsation. It is not, however, so much the displacement of the heart downwards as a whole that is important in the diagnosis of aortic aneurysm as the transverse position which the organ takes up in many cases of this disease. Indeed, the heart as seen with the fluorescent screen occupies nearly a transverse position in the chest in many cases of aneurysm of the aortic arch. This position of the organ tends to raise rather than to lower the apex unless there be considerable hypertrophy of the left ventricle, which is quite the exception in aortic aneurysm. No doubt the heart is pushed into this transverse position by the supra-incumbent weight of the aneurysmal sac. I do not think that sufficient stress has been laid on this horizontal position of the heart as a sign of aortic aneurysm. In the absence of other physical signs of aneurysm of the aorta this transverse position of the heart becomes, I think, of great diagnostic value, as the following two cases well illustrate.

CASE 1.-A man, aged 56 years, came to the out-patient room of the City of London Hospital for Diseases of the Chest on Jan. 19th, 1901, with the following history. He had been in good health up to tiree months before when he began

FIG. 1.

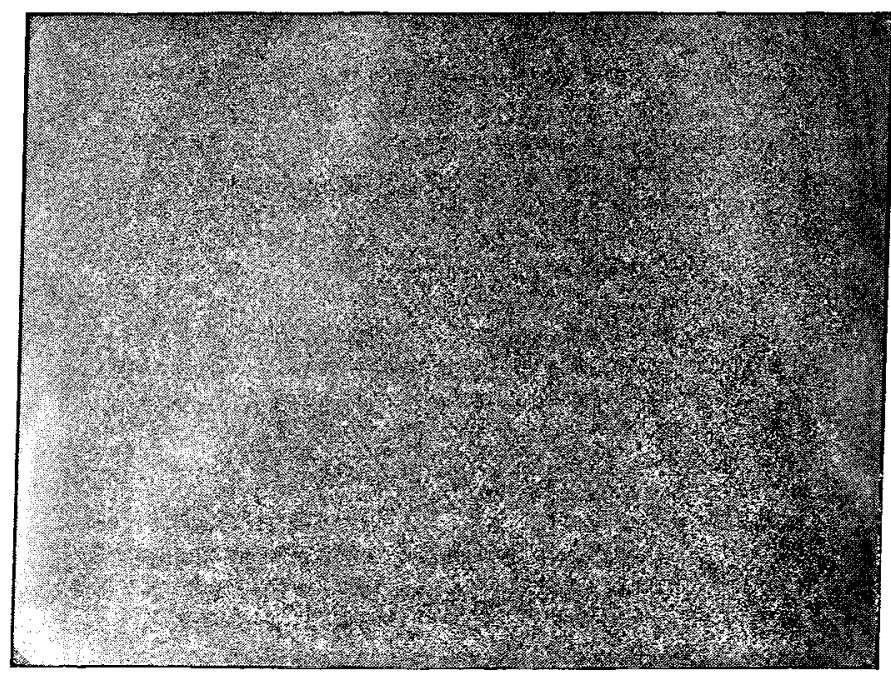

Aneurysm of aorta with transterse position of the heart.

to suffer with dyspncea on exertion. He also complained of pain in the back between the shoulders. He had never had rheumatism and he denied syphilis. On examination he was found to have well-marked signs of aortic regurgitation. But what especially arrested attention was the fact that although the heart's impulse was displaced outwards it was not displaced downvards as we should have expected 
from the amount of regurgitation present as gauged by the pulse, which was typically that of free aortic regurgitation. Tpigastric pulsation was also especially well marked. As far as could be ascertained by palpation and percussion the heart was lying almost transversely across the chest. There were no other physical signs of aortic aneurysm with perhaps the exception of indefinite tracheal tugging. In fact, excepting the position of the heart the case might easily have been passed as one of simple aortic regurgitation. Having noticed, however, with the screen this horizontal position of the heart in a previous case of aortic aneurysm, I examined the patient with the screen and saw at once that he had an aneurysm of the aorta. Fig. 1 shows well the transverse position of the heart and aneurysmal sac.

CASE 2.-A man, aged 46 years, came to the outmpatient room at the hospital complaining of cough and dyspncea of three years' duration. The cough was dry and noisy, but it was not typical of an aortic aneurysm. He also complained of some pain in the back. He had never had rheumatism and he denied syphilis. The heart occupied the same tranverse position as in the former case. The heart sounds were clear in all areas, but the aortic second sound was very loud and ringing and was heard over a wide area. The pupils were equal. The pulses were equal and were soft and compressible. The vocal cords acted well and there was no tracheal tugging. The urine was acid, with a specific gravity of 1020 ; there was no albumin. On examination with the screen an aneurysm was discovered, probably of the descending arch. Fig. 2 shows the transverse position of the heart and aneurysmal sac.

\section{FIG. 2}

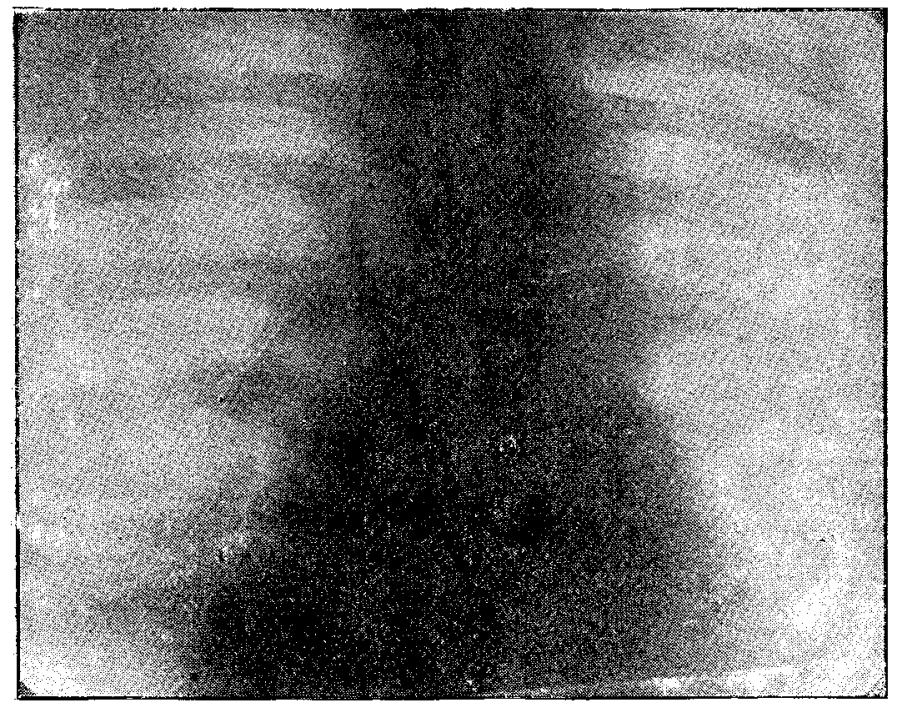

Aneurysm of aorta with transverse position of the heart.

I have ventured to put these two cases on record as illustrating the diagnostic value of this transverse position of the heart in aortic aneurysm.

Harley-street, W.

\section{Clinital Plotes:}

\section{MED ICAL, SURGICAL, OBSTETRICAL, AND THERAPEUTICAL.}

\section{AMPUTATION OF THE THIGH IN AN OCTOGENARIAN.} By John Mưray, M B., C.M. GLasf.

I was called to see the patient, a hale old man, aged 82 years, on Sept. 23rd, 1902, and found him then complaining of shooting pain of an agonising character in the great toe of his right foot. It had commenced a week or two previously and had become steadily worse. The toe was blanched and colder than its fellow on the other foot and sensation was impaired. There was no sign of injury. The pulsation in the posterior tibial was slight and the artery could be felt as a hard cord. The patient had led an active, temperate life and had had practically no illness until 12 years before when he suffered from hæmopericardium. There was marked atheroma of the right radial artery and the vessels in the temporal and frontal regions. The heart was feeble and there was a systolic murmur at the apex. The urine contained no sugar and no albumin. Otherwise his general condition was good. The diagnosis of senile gangrene from atheroma was made and expectant treatment was adopted. The foot was cleansed, wrapped in cotton wool, and kept warm ; opium and digitalis were administered. In course of time the disease progressed till it involved the whole foot although no line of demarcation appeared. The pain was very severe and could not be controlled by free administration of opium.

Operation was at first refused but on Nov. 10th chloroform was administered by Mr. J. Morgan Evans and, assisted by Dr. A. Miller Kerr, I amputated in the lower third of the thigh. The popliteal artery was decidedly atheromatous, but the ligature held securely. The flaps (long anterior and short posterior) were united by a continuous suture for the sake of speed as the patient was looking very ill. Three pints of hot saline fluid were injected subcutaneously with a marked effect upon the pulse and one-third of a grain of strychnine was given hypodermically. During the anæsthesia the patient's breathing was of the Cheyne-Stokes character and continued so for three days. The cycle of breathing was interesting: on the day of operation the period of respiration was 20 seconds, with 40 seconds apncea, on the second day the periods were 30 seconds each, and on the third day the period of respiration was 40 seconds and that of apncea was 20 seconds. Afterwards it gradually improved. Water was the only thing which the patient would take for 24 hours after operation and of that he had as much as he would drink; later he took nourishment well and made an excellent recovery.

At the age of 82 years amputation of the thigh is an uncommon operation and it is unlikely that $I$ would have operated had it not been for the strong desire of the patient to have something done which would relieve him. At the present date-five months after the operation-the stump is perfectly sound. It may be added that just the other day he had a cerebral attack causing aphasia, no doubt due to a thrombosis in one of the vessels in his left frontal convolution.

Llandrindod Wells.

\section{AN ERROR IN THE ESTIMATION OF THE SPECIFIO GRAVITY OF THE BLOOD BY HAMMERSCHLAG'S METHOD.}

\section{By A. G. Levy, M.D. Lond.}

HAMMERSCHLAG's method being rapid and convenient is the one most generally employed clinically, but it is subject to an error of varying magnitude. This error consists of an excessive reading and is found to be caused by the disturbing effect upon the hydrometer of the low surface tension of the mixture of chloroform and benzol employed. The small hydrometers commonly used for this purpose are most affected and in one instrument so large a discrepancy as 14 degrees of the scale was noted; more usually, however, the error varies from three to ten degrees.

It is well to employ one of the following methods to obviate the inaccuracy.

1. The estimation of the specific gravity of the chloroform and benzol mixture by means of an instrument which excludes or minimises the surface tension factors. The most convenient is some such balance as Westphal's, in which the surface of the fluid is intersected by an exceedingly fine platinum wire only. The employment of specific gravity beads is inconvenient on account of the long series required. The picnometer method is not readily applicable in connexion with very volatile fluids.

2. By employing a hydrometer which has been standardised or corrected in chloroform and benzol mixtures, the requisite specific gravities of which have been adjusted by an accurate method. In the absence of the above-mentioned appliances a rough method of correction may be applied to any hydrometer of which the highest mark is $1 \cdot 000$. The method consists of adjusting the proportions of a mixture of chloroform and benzol until a small drop of water immersed

1 Abstract of a paper read before the Royal Society in December, 1902. 